\begin{tabular}{c|l|l|l} 
Case Reports $h$ & Case Rep Dermatol 2010;2:110-115 & Published online: July 2, 2010 & $\begin{array}{l}\text { @ 2010 S. Karger AG, Basel } \\
\text { ISSN 1662-6567 } \\
\text { Www.karger.com/cde }\end{array}$ \\
\hline
\end{tabular}

\title{
A Case of Orbital Xanthogranuloma Treated by Surgical Excision
}

\author{
Fumiaki Shimizu $^{\mathrm{a}}$ Hiroko Taneda $^{\mathrm{a}}$ Haruaki Sato $^{\mathrm{a}}$ \\ Aiko Kato $^{\mathrm{a}}$ Naoko Takeo ${ }^{\mathrm{b}}$ Sakuhei Fujiwara ${ }^{\mathrm{b}}$

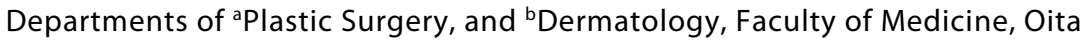 \\ University, Yufu, Japan
}

\section{Key Words}

Xanthogranuloma $\cdot$ Orbital region $\cdot$ Surgical treatment

\begin{abstract}
Orbital xanthogranuloma is an uncommon tumor. It is usually associated with a systemic or hematological disease. This report presents a rare case of orbital xanthogranuloma associated with heart disease and thrombocytopenia. A 52-year-old female presented with a bilateral periorbital subcutaneous tumor that had existed for 3 years. Although immunoglobulin levels were within the normal limits, thrombocytopenia, slight anemia and increased levels of C-reactive protein and alkaline phosphatase were observed. The mass was excised successfully. The malar flap elevation technique made it easy to approach the periorbital subcutaneous mass. A histopathological study led to a diagnosis of xanthogranuloma based on the presence of infiltration of histiocytes and Touton-type giant cells.
\end{abstract}

\section{Introduction}

Orbital xanthogranuloma is a rare entity, which usually presents as bilateral diffuse or nodular lesions in the eyelid or orbit [1]. Such lesions may be associated with a systemic disease such as hyperlipidemias, diabetes mellitus, blood abnormality including IgG monoclonal gammopathy, plasmacytosis, cryoglobulinemia, chronic lymphocytic leukemia or asthma and therefore necessitate a thorough systemic evaluation [1-6]. Periorbital lesions are treated with chemotherapy, radiotherapy, steroidal injection, or local excision [1-6]. Furthermore, due to the rarity of this disease, the treatment of this condition is largely anecdotal and with variable results. Though local excision is successful in some cases [3], surgical debulking of the lesion leads to recurrence in other cases [5]. There is no report that describes details of the surgical approach to the 


\begin{tabular}{l|l|l|l} 
Case Reports $\boldsymbol{h}$ & $\begin{array}{l}\text { Case Rep Dermatol 2010;2:110-115 } \\
\text { D01: } 10.1159 / 000316807\end{array}$ & Published online: July 2, 2010 & $\begin{array}{l}\text { O 2010 S. Karger AG, Basel } \\
\text { ISSN 1662-6567 } \\
\text { www.karger.com/cde }\end{array}$ \\
\hline
\end{tabular}

periorbital lesion in this disease. This report presents a case of orbital xanthogranuloma accompanied by thrombocytopenia and slight anemia treated by surgical excision.

\section{Case Report}

A 52-year-old female presented with painless protrusion of a bilateral subcutaneous facial mass that had gradually progressed for 3 years. She visited a plastic surgeon, and a part of the tumor of her right lower eyelid was resected; however, the pathological diagnosis was not determined. The surface of her skin looked normal. Unmovable bilateral hard masses extended from the lateral canthus to the lower eyelids and one hard mass was observed in the glabellar region (fig. 1a). MRI showed a poorly-defined mass, which was hypointense on T1 and T2 weighted imaging, on the periorbital bone (fig. 2). A systemic examination did not reveal any abnormality of the internal organs. Although the serum levels of IgG, $\operatorname{IgM}$ and IgA were within normal limits, thrombocytopenia $\left(6-11 \times 10^{4} / \mathrm{mm}^{3}\right)$, slight anemia $\left(3.3-3.7 \times 10^{5} / \mathrm{mm}^{3}\right)$ and an increased level of C-reactive protein $(1.07-1.73 \mathrm{mg} / \mathrm{dl}$; normal) and alkaline phosphatase (371-537 IU/dl; normal) were observed. The heart ultrasound examination showed a normal ejection function even though the patient had received two cardioarterial by-pass grafts due to unstable angina pectoris. The earlier specimen which was taken by the plastic surgeon was reexamined, and a tentative diagnosis of juvenile/infantile myofibromatosis was made. The patient underwent tumor excision. A skin incision was made along the line from the lower lid margin to the cheek. The skin flap was elevated above the superficial muscular aponeurotic system and the masses were exposed below this skin flap. This approach was similar to the malar flap elevation and the mass was excised without any safety margin (fig. 1b). The skin above the mass was preserved. The subcutaneous tumor around midline was preserved because the mass did not show rapid growth and the patient did not desire extensive surgery. Histopathology revealed the infiltration of histiocytes, lymphocytes, and plasma cells within increased collagen bundles and periorbital muscles. Lymphocytes were aggregated like lymphoid follicles with or without germinal centers in various fields. Some areas showed large cells with foamy cytoplasm and Touton-type multinucleated giant cells with a wreath-like arrangement of nuclei and cytoplasmic vacuoles around the nuclei (fig. $3 \mathrm{a}$ ). There was no evidence of necrobiosis. Immunological staining revealed that CD68-positive and S-100-negative cells were increased in the tumor (fig. 3b). Based on these features, the final diagnosis was orbital xanthogranuloma. The tumors were resected without measurable excision margins. The patient did not show any sign of regrowth of the mass or

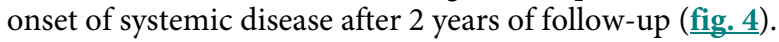

\section{Discussion}

Orbital xanthogranuloma was first reported in 1991 [4] and was categorized as a nonLangerhans histiocytic disorder. Eighteen more cases have been reported subsequently [5]. Xanthogranulomas show a characteristic histopathological appearance. There is a proliferation of histiocytes, plasma cells and lymphocytes with Touton giant cells that stain positive for lipid. Touton giant cells are multinucleated cells with the nuclei arranged in a wreath around a nidus of eosinophilic cytoplasm and separated from the cell membrane by a rim of translucent foamy cytoplasm [2] .

Although it is unclear whether the etiology of this disease is a tumor or immune reaction, a concomitant immunological abnormality has been suggested. Bullock et al. suggested that serum immunoglobulins were complexed with lipids and deposited in the skin, eliciting a foreign-body giant-cell reaction [6].

Orbital xanthogranuloma is a clinical diagnosis with histological support. The exclusion of other xanthogranulomatous conditions is therefore of prime importance in its management. Necrobiotic xanthogranuloma shares some clinical and histological similarities with orbital xanthogranuloma [1]. Tang et al. described that it was possible that necrobiotic xanthogranuloma is a disease spectrum with orbital xanthogranuloma being a mild form $[1]$. 
The periorbital lesions should be differentiated from juvenile or adult type xanthogranuloma. Orbital xanthogranuloma is much more extensive with frequent involvement of the subcutis, whereas the juvenile or adult type xanthogranuloma is a well circumscribed dermal lesion [3].

Erdheim-Chester disease is the association of adult-onset xanthogranulomas with the long bones and some systemic disease, especially with diffuse sclerosis of diaphysis and metaphysis of the long bones and sometimes with hepatosplenomegaly and hepatic adenomas [1-3]. Ocular involvement is usually rare. In the current case, no hepatic or splenic involvement was observed by systemic CT scanning.

Nearly all of the cases reported in the literature have abnormal blood tests and/or an associated systemic disease. The current case is unique because there was a massive, bilateral tumor associated with heart disease and thrombocytopenia, slight anemia, and an increased level of C-reactive protein and alkaline phosphatase. The significance of these latter two abnormalities is not known. One case of orbital xanthogranulomas associated with thrombocytopenia is reported [1].

In cases where the periorbital mass causes an optic problem due to intraorbital compression or a cosmetic problem, some form of treatment is necessary to remove the mass. Various treatment modalities have been tried including local excision, radiotherapy, periocular and systemic steroids, chemotherapy, radiotherapy and plasmapheresis [1-6]. The effect of surgical debulking is thought to be limited because of recurrence within 6 months to 1 year [5]; local excision is performed when conservative treatment such as steroids treatment or radiotherapy is not effective. There are no reports describing a detailed surgical approach to excise a periorbital mass. In this case, the malar flap elevation technique was applied. This approach made it easy to excise the periorbital mass. Furthermore, the scar was not obvious. A similar approach is performed for sentinel lymph node biopsy in patients with periocular skin malignancies [7]. The disadvantage of this approach is that it is difficult to excise the mass around mid-line. When the mass invades the skin, the skin cannot be preserved, and an obvious scar will remain. However, this skin defect can be reconstructed with a malar flap transfer.

Adjuvant therapy was not conducted following the excision. However, in one previous report, paraproteinemia appeared 25 years after the onset of orbital xanthogranuloma [5]. Therefore, basic screening for systemic disease and a long-term follow-up will be necessary in this case. 
Fig. 1. a The patient presented with a gradually progressive protrusion of a bilateral subcutaneous mass. The surface of the skin looked normal. There was an unmovable hard mass around the orbit and one hard mass in the glabellar region. Black arrows indicate a subcutaneous mass. b Skin incision was made along the line from lower lid margin to the cheek. This approach was almost fitted to the technique of so-called malar flap elevation. Solid lines indicate skin incision and broken lines indicate subcutaneous mass, which was resected completely in this approach.
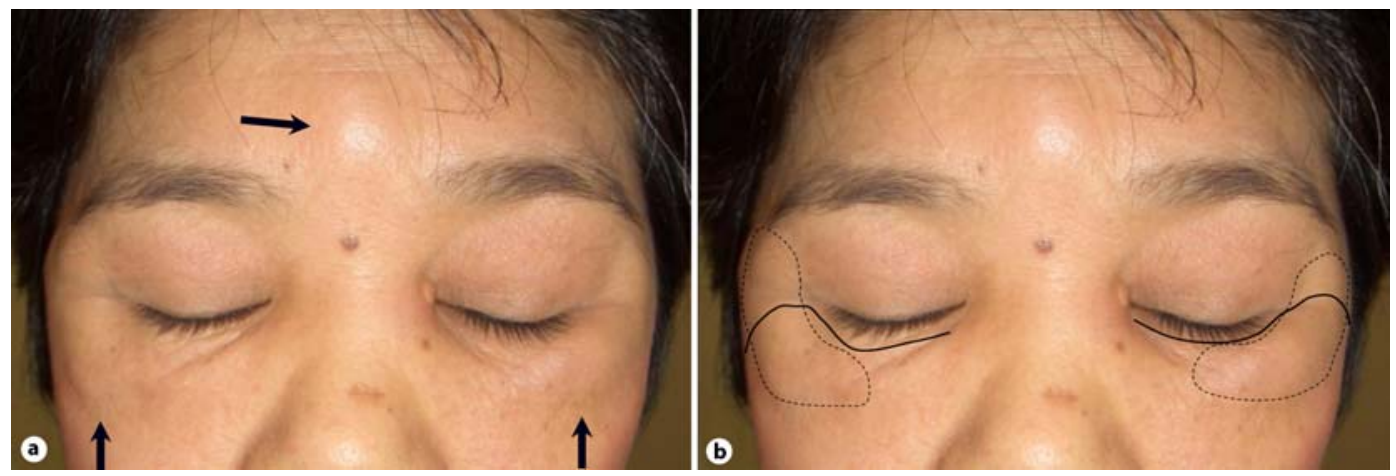

Fig. 2. MRI showed a poorly-defined mass, which was hypointense in this T1 and T2 weighted image, on the periorbital bone.

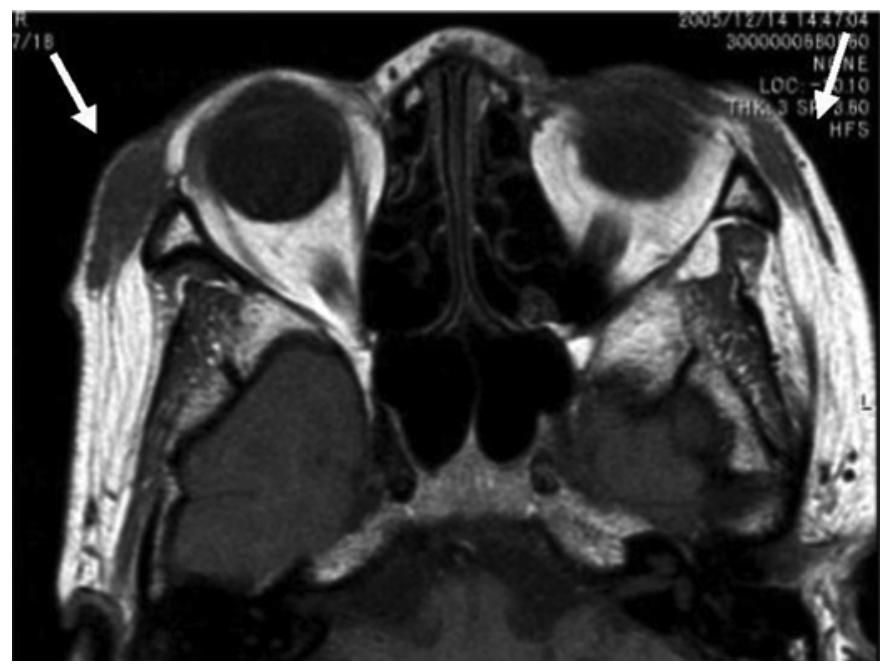


Fig. 3. a Histological appearance of the periorbital mass, HE stain. A dense collagenous tissue with inflammatory infiltrates was observed: the black arrow indicates Touton-type giant cells with a wreathlike arrangement of nuclei. There was no evidence of necrobiosis. b Immunological staining revealed CD68-positive cells in the tumor.
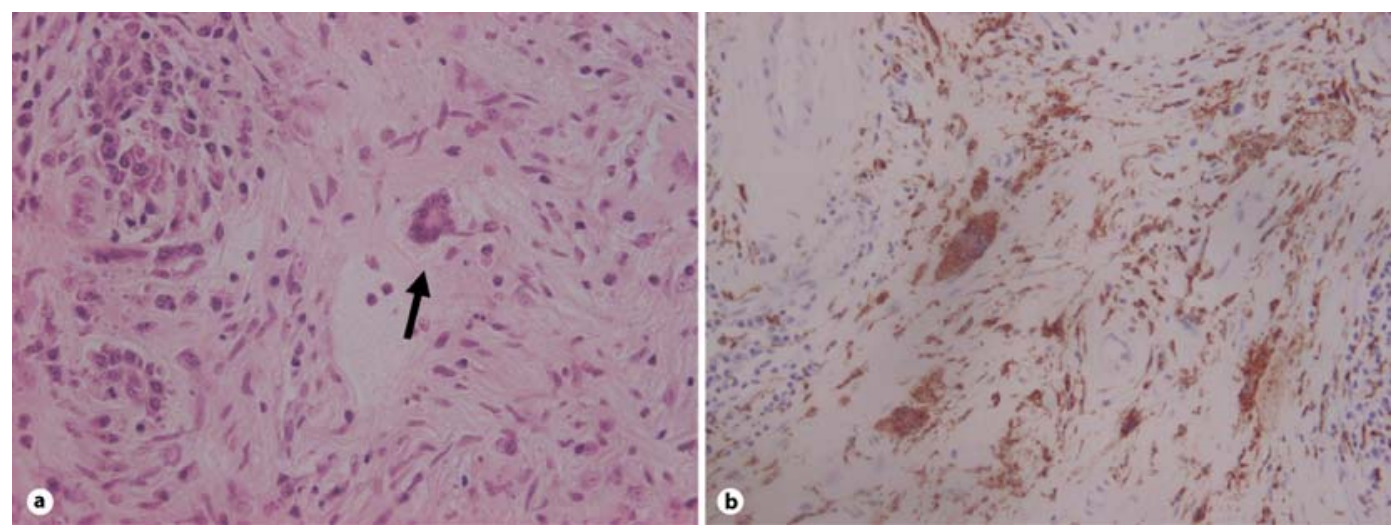

Fig. 4. The patient did not show any sign of regrowth of mass or onset of systemic disease at 2 years' follow-up. The post-operative scar was not obvious.

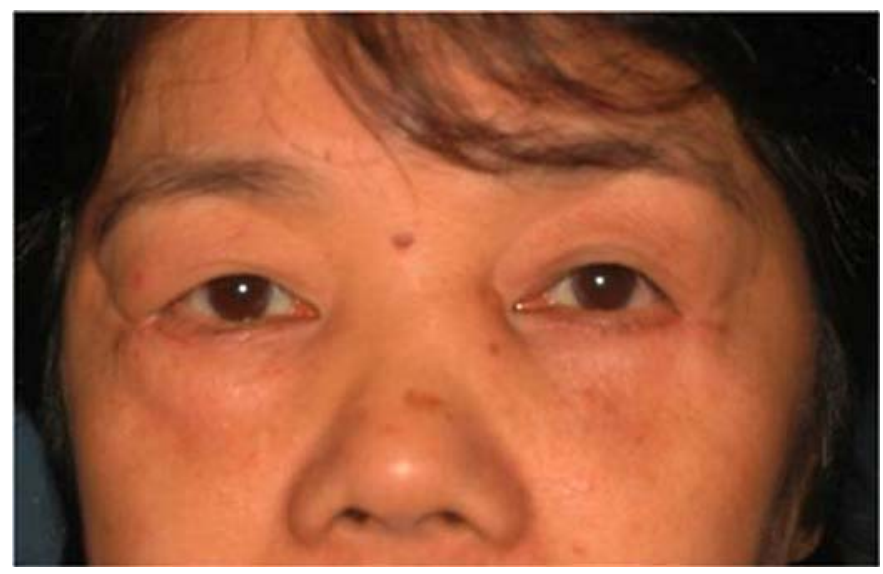




\section{References}

-1 Tang WK, Lee KC, Chong LY: Three patients with orbital xanthogranuloma and non-progressive haematological abnormalities. J Dermatol 2003;44:185-189.

2 Elner VM, Mintz R, Demirci H, and Hassan AS: Local corticosteroid treatment of eyelid orbital xanthogranuloma. Trans Am Ophthalmol Soc 2005;103:69-74.

-3 Ramesh M, Santosh H, Geeta V, Milind N, Sanghamitra B: Isolated giant xanthogranuloma of the orbit. Ind J Ophthalmol 2007;55:156-158.

-4 Rose GE, Patel BC, Garner A, Wright JE: Orbital xanthogranuloma in adult. Br J Ophthalmol 1991;75:680-684.

-5 Jakobiec FA, Mills MD, Hidayat AA, Dallow RL, Townsend DJ, Ault Brinker EA, Charles NC: Periocular xanthogranuloma associated with severe adult onset asthma. Trans Am Ophthalmol Soc 1993;91:99-125.

-6 Bullock JD, Bartley GB, Compbell RJ, Yanes B, Connelly PJ, Funkhouser JW: Necrobiotic xanthogranuloma with paraproteinemia. Case report and pathogenetic theory. Ophthalmol 1986;93:1233-1236.

7 Motomura H, Hatano T, Maruyama Y, Yamanaka K, Harada T: A malar flap incisional approach for sentinel lymphnode biopsy in patients with periocular skin malignancies. J Plast Reconstr Aesthetic Surg 2008;62:e184-e186. 